\title{
INFLUENCE OF THE PREHEATING LAYER CHARACTERISTICS IN A TWO-LAYER POROUS BURNER
}

\author{
T. C. HAYASHI, ${ }^{1}$ I. MALICO, ${ }^{2 *}$ and J. C. F. PEREIRA ${ }^{3}$ \\ ${ }^{1}$ Department of Chemical Engineering, Universidade Federal da Bahia, \\ R. Aristides Novis, 2, Federação, 40210-630, Salvador, BA, Brazil \\ ${ }^{2}$ Department of Physics, University of Évora, R, Romão Ramalho, 59, \\ 7000-671, Évora, Portugal \\ ${ }^{3}$ Department of Mechanical Engineering, Instituto Superior Técnico, \\ Av. Rovisco Pais, 1049-001 Lisbon, Portugal
}

\begin{abstract}
The influence of the perforated plate characteristics that precedes the flame support layer in a newly designed bilayered porous combustor was numerically assessed. It is shown that a perforated plate with $1 \mathrm{~mm}$ diameter holes is able to provide the necessary quenching of the reaction front, leading to the stabilization of the flame within the pores of the flame support layer for all the range of operating conditions and is, thus, an appropriate choice. The influence of the diameter of the holes on the gas and solid temperatures, and on the emissions of pollutants, namely NO and $\mathrm{CO}$, is discussed.
\end{abstract}

Keywords: Porous burners; CFD; Flame stability; Modeling

\section{INTRODUCTION}

Porous burners are characterized by the stabilization of a flame front inside a porous matrix and, owing to an internal feedback of heat, present several advantages when compared to conventional unconfined flame burners. Good reviews of the theoretical background and experimental/numerical studies on combustion in porous media can be found, for example, in Howell, Hall, and Ellzey (1996), Viskanta and Gore (2000), and Durst and Trimis (2002).

Several geometrical configurations of porous burners have been proposed (e.g., Kulkarni and Peck, 1992; Howell et al., 1996; Hsu, Evans, and Howell, 1993; Mohammad, Ramadhyani, and Viskanta, 1994; Durst and Trimis, 2002), being the focus of this study on two-layer porous burners. This type of porous burner is composed of two porous layers with different porosities and, possibly, of different materials. The flame front is stabilized within the higher-porosity

\footnotetext{
*Address all correspondence to Prof. Isabel Malico, imbm@uevora.pt
} 
layer, the flame support layer, which is preceded by another layer with pores of smaller diameter, called the diffuser layer. In these burners, stabilization of the flame front for a broad range of operating conditions is achieved due to the abrupt change in the heat transfer properties of each layer.

Kulkarni and Peck (1992) (in Howell et al., 1996) is possibly one of the first studies on this type of burner. In this work the authors considered conductive, convective, and radiative heat transfer, along with a multistep reaction mechanism for methane-air combustion, in a one-dimensional laminar model of a premixed flame. They found that a significant improvement in the radiative output of the burner could be attained by optimizing the properties of the materials upstream and downstream of the flame front. Generally, the upstream (diffuser) layer should be of lower porosity, shorter length, and higher optical thickness than the downstream (flame support) layer. Moreover, the diffuser layer should be highly scattering and the flame support layer nonscattering.

A porous burner composed of two porous ceramic cylinders was experimentally tested and numerically simulated by Hsu et al. (1993). Among other results, it was shown that it is possible to control the preheating effect by using ceramics of different porosities in the diffuser layer. It was observed that as the extinction coefficient of the material of the diffusive layer increases, the amount of preheating is reduced, as are the peak flame temperatures and the temperatures downstream of the flame front.

According to Diamantis, Mastorakos, and Goussis (2002), flames stabilized in a two-layer burner present hybrid behavior between single-layer surface flames and single-layer submerged flames. By means of a one-dimensional laminar premixed model for methane-air combustion in porous media, in which the radiation transfer equation is solved inside the porous media, it was shown that the flame support layer acts like the foam downstream of the reaction front in a submerged flame burner, absorbing heat from the hot products and redistributing it mainly forward to the environment, which increases the burner radiant efficiency. Only a small part of the heat absorbed downstream of the flame front is directed toward the layer situated upstream of the reaction zone because of the blockage effect of the small-pore diffuser layer, which absorbs radiation at the interface to help with the flame stability.

A one-dimensional time-dependent model was used by Barra et al. (2003) to study the effects of material properties on flame stabilization in a two-section porous burner. The effects of solid conductivity, the volumetric heat transfer coefficient, and the radiative heat transfer coefficient were examined. It was concluded that the material properties of the solid matrix significantly affect the stable operating range. Heat recirculation in burners of different lengths was quantified by 
Barra and Ellzey (2004), but it was shown that this parameter does not significantly affect the amount of heat recirculated.

This article focuses on an innovative porous burner (Brehmer et al., 2003), in which the functions of the diffuser layer are performed by a perforated plate made of an insulating material. This plate must accomplish the same tasks of a foamy layer (used, e.g., by Hsu et al. (1993), Diamantis et al. (2002), Barra et al. (2003), and Barra and Ellzey (2004)), while not imposing a too high pressure drop in the flow. Thus it is advisable to have the diameter of the holes as big as possible. Early tests of a prototype of the first configuration of the porous burner indicated that a too high temperature was attained at the interface of the two ceramic layers, which resulted in the perforated plate being damaged (Trimis and Glass, 2000). This situation brought about the need to investigate the characteristics of the interface and the ability of the perforated plate to avoid the occurrence of flashback and the transfer of heat upstream to the incoming fuel-air mixture. This study was conducted by means of a three-dimensional model of the porous burner since a detailed study of the flow and heat transfer at the interface of the two layers was aimed for, and one- and two-dimensional models of the burner are not able to cope with the problem. The model includes the NavierStokes equations, the gas and solid energy balances, and the transport equations for chemical species and was computationally implemented using the finite volume/finite differences method. Radiation heat transfer in the solid matrices and local thermal nonequilibrium are accounted for. Additionally, a detailed reaction mechanism is applied to model the combustion process, allowing for the prediction of pollutant formation.

Calculations of the burner using perforated plates with holes of different sizes were conducted. The influence of the diameter of the holes on the temperatures attained by the gas and solid phases as well as on the emissions of pollutants, namely $\mathrm{NO}$ and $\mathrm{CO}$, is discussed.

\section{BURNER}

In Figure 1 a sketch of the burner under study is presented, in which the two ceramic layers are shown. The flame support layer is a thin cylindrical plate of silicon carbide ( $\mathrm{SiC}$ ) foam with 10 pores per inch (ppi) and $90 \%$ porosity. It is preceded by a finely perforated cylindrical plate made of alumina $\left(\mathrm{Al}_{2} \mathrm{O}_{3}\right)$. This burner has been designed and built at the University of Erlangen, Germany, to operate integrated into a newly designed household heating device, fuelled with a biofuel and operating in the power range 2-20 kW (Brehmer et al., 2003). 


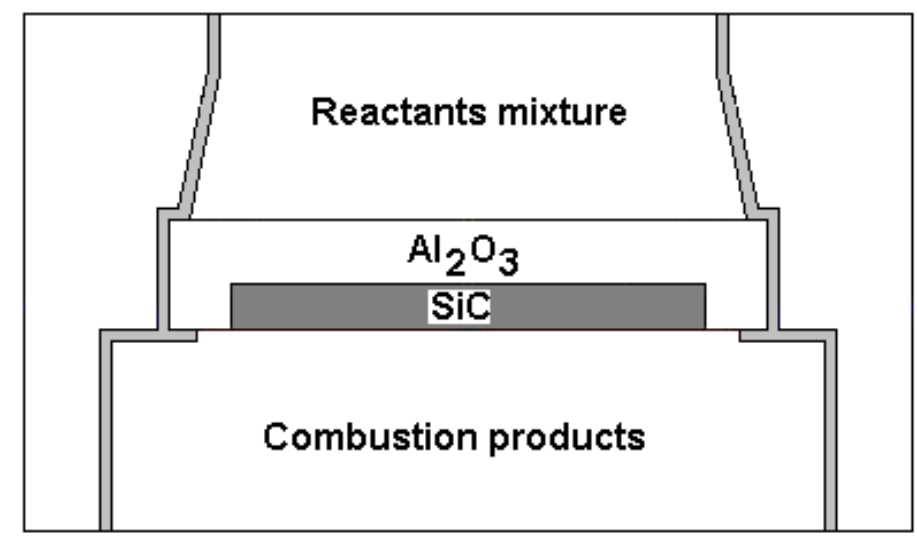

FIGURE 1 Sketch of the burner studied.

The liquid fuel is vaporized in a cool-flame vaporizer and enters the porous burner, premixed with air, at around $500 \mathrm{~K}$. After flowing through the perforated plate, it burns inside the $\mathrm{SiC}$ foam, and the reaction products pass through a highefficiency multijet heat exchanger. This design results in compact, highly efficient equipment, suitable to operate at a large turndown ratio.

\section{MODEL}

To describe the three-dimensional processes taking place inside and downstream of the perforated plate, a three-dimensional model is required. To accomplish this at a reasonable computational cost, a representative fraction of the actual geometry was considered. The volume of the computational domain corresponds to a $1 / 2463$ fraction of the volume of the combustion chamber.

The model unit cell is schematically shown in Figure 2. To simplify the problem, the holes were replaced by square ducts with the same cross-sectional area. In this approach, the actual flow path is modeled in the perforated plate, while the $\mathrm{SiC}$ foam layer is modeled as a continuum with volume-averaged properties.

The set of governing equations includes the steady forms of the volume-averaged macroscopic three-dimensional Navier-Stokes equations along with energy balances for the solid and fluid phases, to allow for the occurrence of local thermal nonequilibrium, and the mass balances of chemical species [see, e.g., (Whitaker, 1969 or Gray, 1975 for details on the averaging procedure]. These read as shown below: 


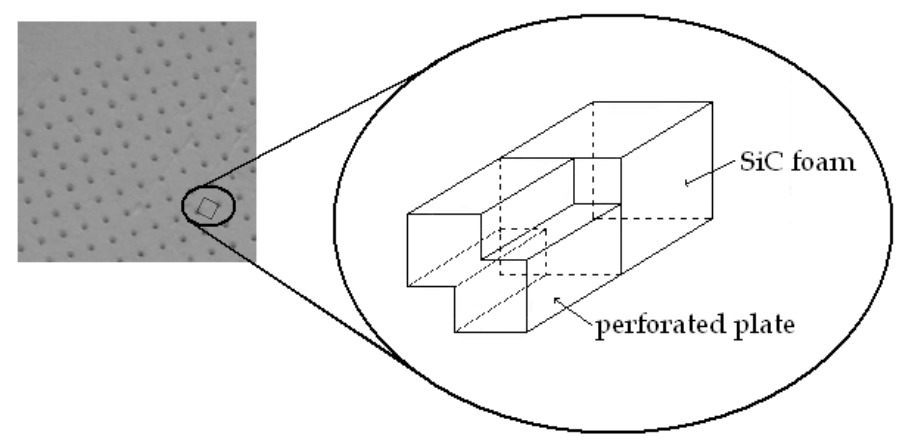

FIGURE 2 Schematic of the representative unit cell.

Continuity equation:

$$
\nabla \cdot\left(\varepsilon \rho_{f} \mathbf{v}\right)=0
$$

Momentum equation:

$$
\nabla \cdot\left(\varepsilon \rho_{f} \mathbf{v} \mathbf{v}\right)=-\varepsilon \nabla p+\nabla \cdot\left(\varepsilon \mu_{f} \nabla \mathbf{v}\right)-(\nabla p)_{p}
$$

Energy balance of the gas phase:

$$
\nabla \cdot\left(\varepsilon \mathbf{v} \rho_{f} c_{p, f} T_{f}\right)=\nabla \cdot\left(\varepsilon k_{f} \cdot \nabla T_{f}\right)+h_{v}\left(T_{s}-T_{f}\right)-\varepsilon \sum_{k=1}^{N_{s p}} \dot{\omega}_{k} M_{k} h_{k}
$$

Energy balance of the solid phase:

$$
\nabla \cdot\left[(1-\varepsilon) k_{s} \nabla T_{s}\right]-h_{v}\left(T_{s}-T_{f}\right)-\left(\nabla \cdot \mathbf{q}_{r}\right)_{p}=0
$$

Mass balance of chemical species:

$$
\nabla \cdot\left(\varepsilon \mathbf{v} \rho_{f} y_{k}\right)=\nabla \cdot\left(\varepsilon \rho_{f} \mathcal{D}_{\mathbf{k m}} \cdot \nabla y_{k}\right)+\varepsilon \dot{\omega}_{k} M_{k} \quad k=1, \ldots, N_{s p}-1
$$

In equations (1)-(5), all symbols are as indicated in the Nomenclature.

The unknown quantities in the governing equations must be estimated by means of closure models. The pressure loss owing to the presence of the porous matrix is not considered in the perforated plate since, in this layer, direct calculation of the flow pathways is performed. In the $\mathrm{SiC}$ foam layer this term is calculated by means of the Ergun equation as modified by Macdonald et al. (1979). 
The net radiative flux within the $\mathrm{SiC}$ foam is obtained by solving the radiative transfer equation with the discrete ordinates method [see, e.g., (Fiveland, 1988) for details on this method]. The medium is assumed to be gray and isotropically scattering, with properties taken from Mital, Gore, and Viskanta (1996). Owing to the much higher emissivity of the solid when compared to the gas, gas radiation is not considered in the $\mathrm{SiC}$ foam. In the perforated plate the gas is also considered as nonparticipating, and the radiative fluxes are imposed on the opaque walls of the holes, which have an emissivity taken from Modest (1993).

The convective heat transfer coefficient for flow within the $\mathrm{SiC}$ foam was taken from Tong and Sathe (1991). For the perforated plate a value of the superficial heat transfer coefficient for the flow inside the holes has been estimated, which was based on developing flow inside a duct at an average velocity in the range of the operating conditions considered (Özişic, 1985).

Oxidation of heptane is modeled by means of a skeletal mechanism (43 reactions involving 26 chemical species) based on the model of Seshadri, Bollig, and Peters (1997), which was modified, following Tseng (1995), to account for the formation of nitrogen compounds.

Detailed calculation of the thermophysical and transport properties of the reacting mixture is accomplished by making use of the CHEMKIN code package for analysis of gas-phase chemical kinetics (Kee, Rupley, and Miller, 1995a) along with the TRANFIT code package for evaluation of gas-phase multicomponent transport properties (Kee et al., 1995b).

As boundary conditions, the fluid velocity, temperature, and composition are prescribed at the inlet section of the combustion chamber. A zero-gradient condition is assumed for all quantities in the far-field outlet boundary. Moreover, the temperatures of the inlet and outlet planes of the solid layers are calculated, accounting for the radiation heat transfer with imaginary solid walls placed upstream and downstream of the calculation domain, to include the interaction of the combustion chamber with the other components of the heating system. Symmetry conditions are imposed across the $y$ - and $z$-oriented boundary planes parallel to the main flow direction. The nonslipping condition is imposed at the walls of the holes of the perforated plate, which are also treated as impervious to the flux of chemical species.

Equations (1)-(5) were numerically solved by means of the finite volume/finite differences method. The details of the numerical model and of the calculation procedure can be found in Hayashi, Malico, and Pereira (2004). Typical CPU times for a mesh containing 37,268 points varied between 16 and 85 hours on i686 Intel Pentium 4 processors with $2.4 \mathrm{GHz}$ clocks. 


\section{RESULTS}

The combustion chamber was studied numerically. Two perforated plates with holes of different sizes were considered. The holes of the first plate (PPH2) are 2 $\mathrm{mm}$ in diameter, while the second one (PPH1) presents holes $1 \mathrm{~mm}$ in diameter. In both plates the perforations are drilled in a regular arrangement, with the distance between consecutive rows of holes, measured in the centerlines, being equal to $3.5 \mathrm{~mm}$. The numerical model was validated against experimental results for a broad range of operating conditions (Hayashi, 2005).

\section{PPH2}

The burner with the perforated plate with holes $2 \mathrm{~mm}$ in diameter (PPH2) was simulated for a broad range of operating conditions, with power in the range 4-20 $\mathrm{kW}$ and the excess air ratio coefficient ranging between 1.0 and 1.7.

For most of the operating conditions considered the flame front stabilizes in the ceramic foam, near the interface of the two porous layers. However, exceptions to this are observed at the low-power and low excess air ratio conditions, as is shown in Figure 3.

It was expected that the flame temperature would decrease with the increase of dilution for a given thermal load and increase with power for a fixed air ratio

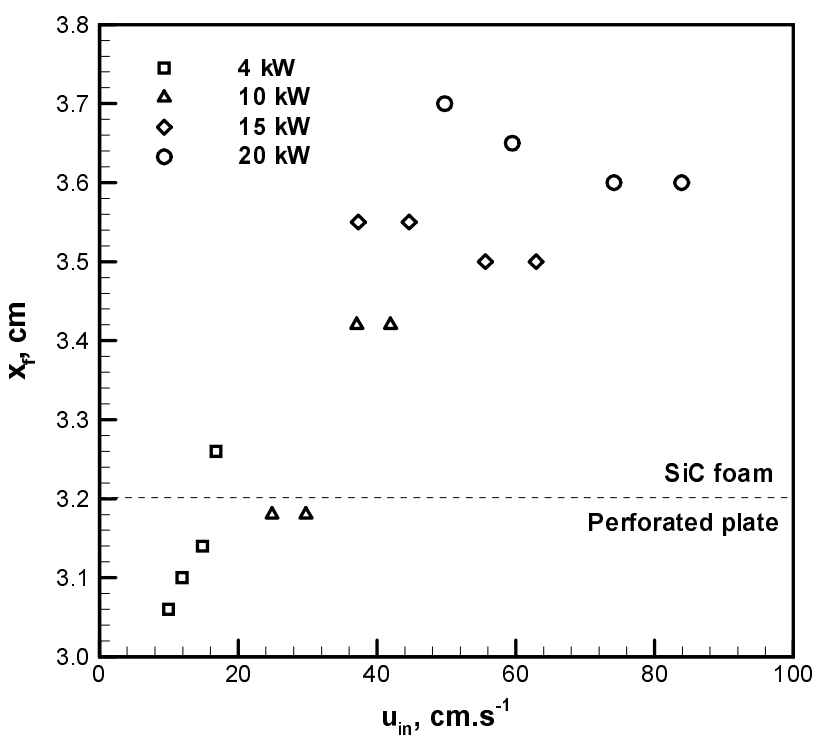

FIGURE 3 Predicted flame position as a function of the inlet flow velocity for the burner operated with perforated plate $\mathrm{PPH} 2$. 


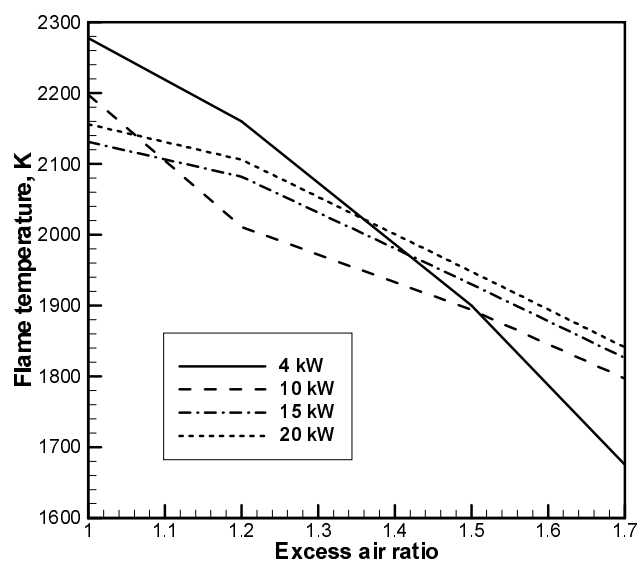

(a)

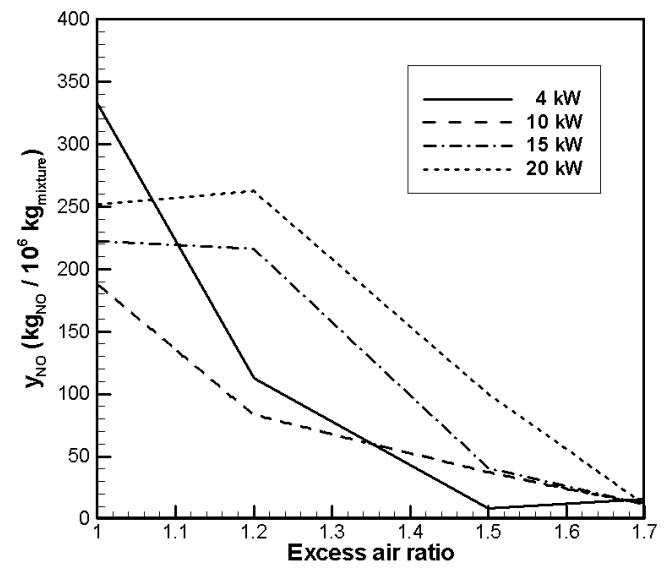

(b)

FIGURE 4 (a) Flame temperature and (b) predicted NO emission as functions of the operating conditions for the burner with perforated plate PPH2.

coefficient. This behavior is observed in Figure 4a for the operating conditions corresponding to higher velocities, but not at $4 \mathrm{~kW}$ and excess air ratios in the range between 1.0 and 1.5 and at $10 \mathrm{~kW}$ with an excess air ratio of 1.0. This unexpected behavior is also noticeable by observing the predicted trend of the NO emission in this burner, which is shown in Figure 4b. The influence of load and dilution on the formation of NO was expected to be the same as on the temperature. 


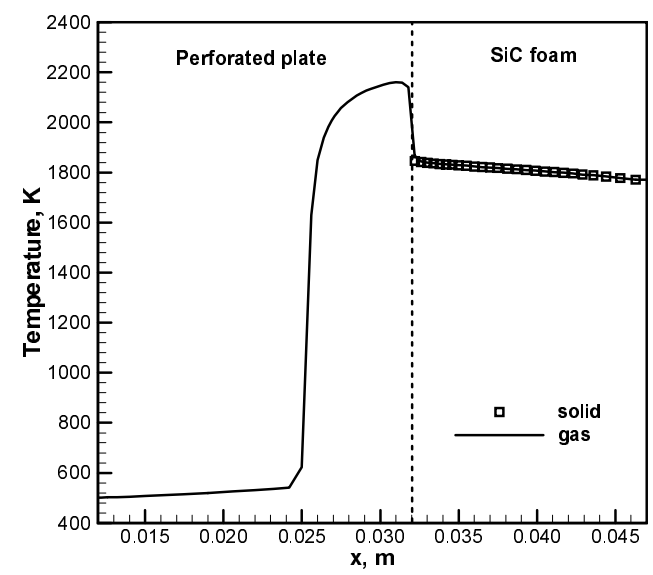

(a)

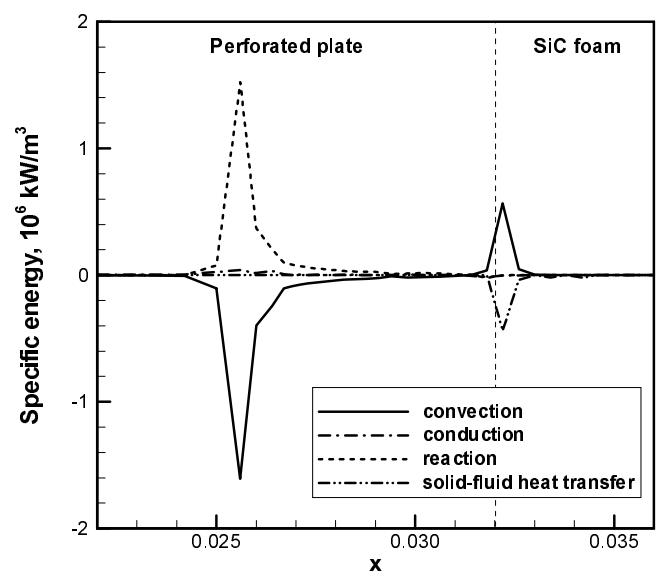

(b)

FIGURE 5 (a) Predicted gas and solid temperature and (b) energy balance of the gas phase along the centerline of a hole for the burner operating at $4 \mathrm{~kW}$ power and $\lambda=1.2$ with the PPH 2 perforated plate.

This behavior in the low velocity flow conditions is due to the aforementioned anchoring of the flame front in the final portion of the holes in the perforated plate, close to the interface between the two layers. The stabilization of the flame front inside the holes is an unwanted situation because it indicates that the holes are larger than they should be to prevent the occurrence of flashback.

To better illustrate these results, the gas and solid temperatures along the centerline of a hole are presented in Figure 5a for the case with $4 \mathrm{~kW}$ and an ex- 
cess air ratio equal to 1.2. The abrupt rise of the gas temperature is observed in the final portion of the holes, in the position where the highest release of heat takes place, as indicated in Figure 5b, which presents the respective gas-phase energy balance. When analyzing this energy balance, again, it can be noticed that the flame shows a different behavior from the typical flames submerged in inert porous media. Because the flame is anchored inside the holes of the perforated plate that serves as the preheating layer, the heat released in the reaction is balanced by convection only since conduction in the gas is very weak.

It is observed that for the lowest inlet velocities and the plate with the larger holes, most of the heat accompanying the chemical reactions is released in the final portion of the holes, yielding a high peak temperature (actually higher than the one observed for the plate with smaller holes; see Figure 9). This leads to a too high temperature at the interface of the two porous media. Confirming this is the fact that the perforated plate was damaged when holes of $2 \mathrm{~mm}$ were used (Trimis and Glass, 2000). It is also shown in Figure 5a that the enhanced heat transfer between the $\mathrm{SiC}$ foam and the fluid results in a fast decrease of the gas temperature as the flow leaves the perforated plate.

\section{PPH1}

The burner with the perforated plate with holes $1 \mathrm{~mm}$ in diameter (PPH1) was simulated under the set of operating conditions for which flashback was predicted for the perforated plate with larger holes (i.e., for power loads of 4 and $10 \mathrm{~kW}$ and an excess air ratio coefficient ranging between 1.0 and 1.5 and from 1.0 to 1.2, respectively). With this plate the flame front stabilizes in the $\mathrm{SiC}$ foam for all the operating conditions considered. This indicates that a reduction of the diameter of the holes of the perforated plate to half the value proposed initially is appropriate to prevent flashback.

For a comparison with the former predictions for the PPH2 plate, the solid and gas temperature profiles along the centerline of a hole are presented in Figure 6 , along with the corresponding gas-phase energy balance, for the burner operating at $4 \mathrm{~kW}$ power and $20 \%$ excess air ratio with the perforated plate with smaller holes. For this plate, preheating of the reactant mixture is mainly due to the convective heat transfer from the solid struts in the $\mathrm{SiC}$ foam, upstream of the reaction front. When the flame stabilizes in the $\mathrm{SiC}$ foam, heat is more efficiently removed from the flame front and transferred to the reactants, resulting in a lower peak temperature of the gas when compared to the case when the flame is anchored inside the holes of the perforated plate. 


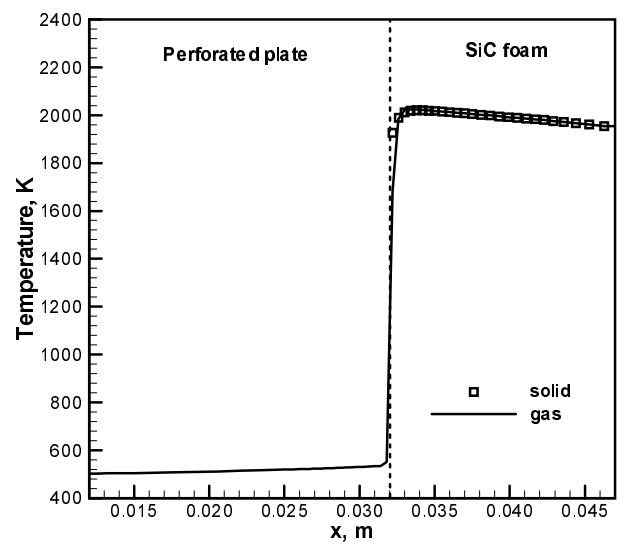

(a)

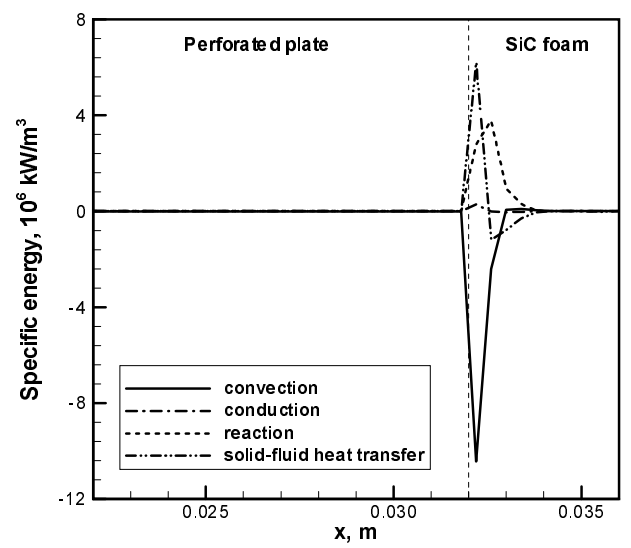

(b)

FIGURE 6 (a) Predicted gas and solid temperature and (b) energy balance of the gas phase along the centerline of a hole for the burner operating at $4 \mathrm{~kW}$ power and $\lambda=1.2$ with the PPH1 perforated plate.

\section{Influence of the Diameter of the Holes of the Perforated Plate on the Performance of the Burner}

The predicted radiant output of the burner as a function of the operating conditions and of the diameter of the holes of the perforated plate is presented in Figure 7. It is observed that owing to the higher temperatures attained in the $\mathrm{SiC}$ foam when the flame front stabilizes in this layer, the radiant output of the burner operating with the plate of smaller holes (PPH1) is higher than when using the plate with holes $2 \mathrm{~mm}$ in diameter. 


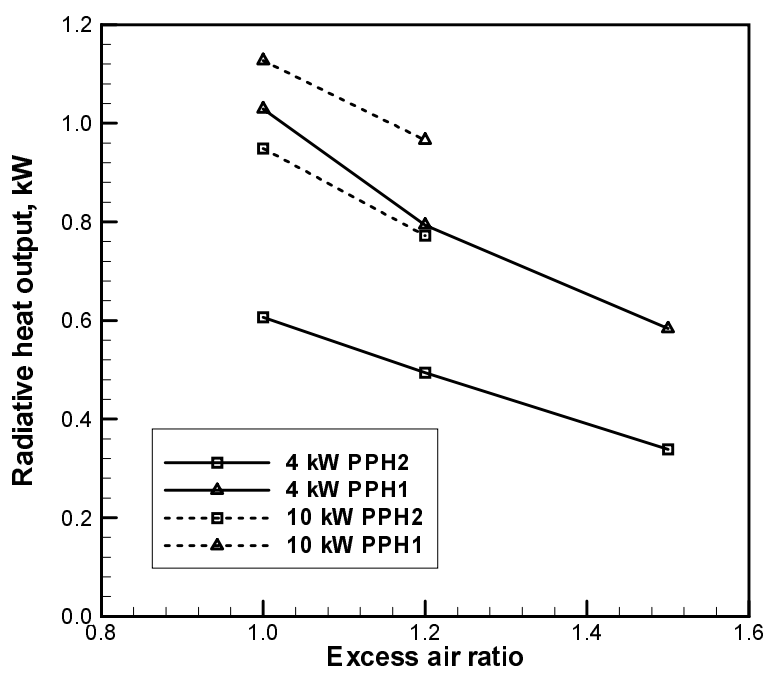

FIGURE 7 Radiant output of the burner as a function of the operating conditions and diameter of the holes of the perforated plate.

Regarding the case in study, heat should be transferred to the product stream in the form of sensible heat. Even though the temperature of the gases leaving the burner increases when the flame is stabilized in the $\mathrm{SiC}$ foam layer, the accompanying increase in the radiant output results in a smaller fraction of the energy output of the burner being carried out with the product stream. This is shown in

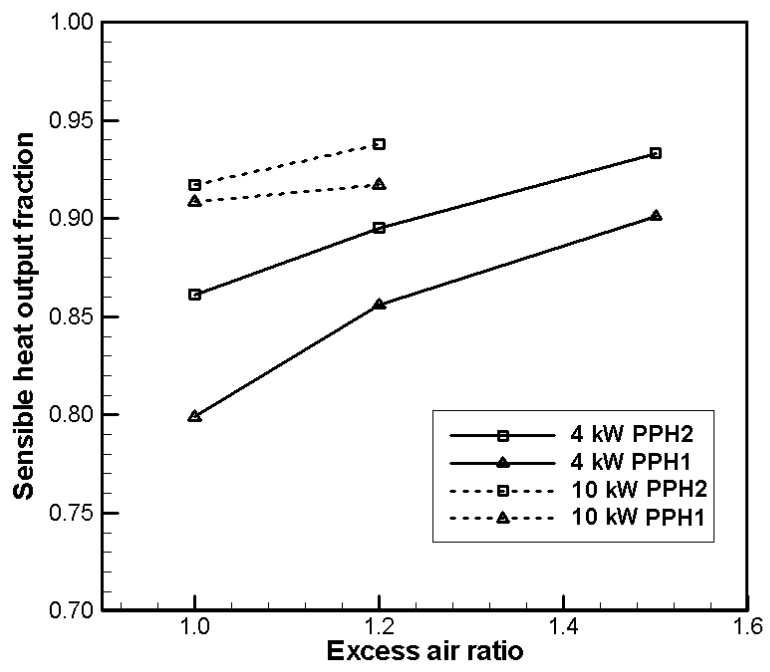

FIGURE 8 Fraction of the output energy from the burner in the form of sensible heat as a function of the operating conditions and diameter of the holes of the perforated plate. 


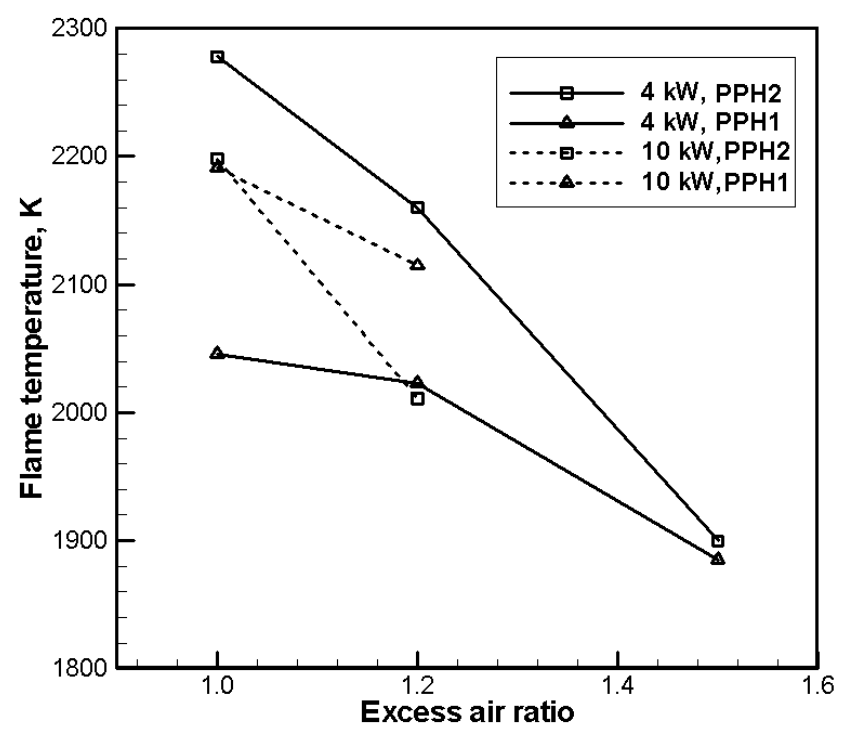

FIGURE 9 Flame temperature as a function of the operating conditions and diameter of the holes of the perforated plate.

Figure 8, which presents the influence of the perforated plate and operating conditions on the fraction of output energy delivered as sensible heat. It is observed that the effect of the hole diameter is less pronounced for the higher $10-\mathrm{kW}$ load cases. This is due to the fact that the increase of the flow speed with the power results in the flame stabilizing closer to the interface of the two layers.

The influence of the diameter of the holes of the perforated plate on the flame temperature is presented in Figure 9 as a function of the operating conditions. The predictions for the burner with the PPH1 plate follow the expected trend; that is, the flame temperature increases with power for a fixed excess air ratio and decreases with the increase in the dilution for a given load.

The influence of the flame position on the NO formation has been also investigated. In Figure 10 a comparison is made of the evolution of NO formation rate along the centerline of the holes of the perforated plate for the burner operating at $4 \mathrm{~kW}$ load and 1.5 excess air ratio and for the two perforated plates considered.

The model considered three pathways for the formation of NO (Hayashi, 2005), however, only two have been plotted in the logarithm graph in an attempt to avoid the superposition of lines. Figure 10 thus shows the contribution of Zeldovich's thermal-NO mechanism and of an $\mathrm{N}_{2} \mathrm{O}$-consuming reaction set, along with the total production rate. The third pathway considered for the formation of nitrogen oxide is a reversible $\mathrm{NO}_{2}$-consuming reaction, equation (6). The NO production in this latter route is important only as an initial step of the NO forma- 


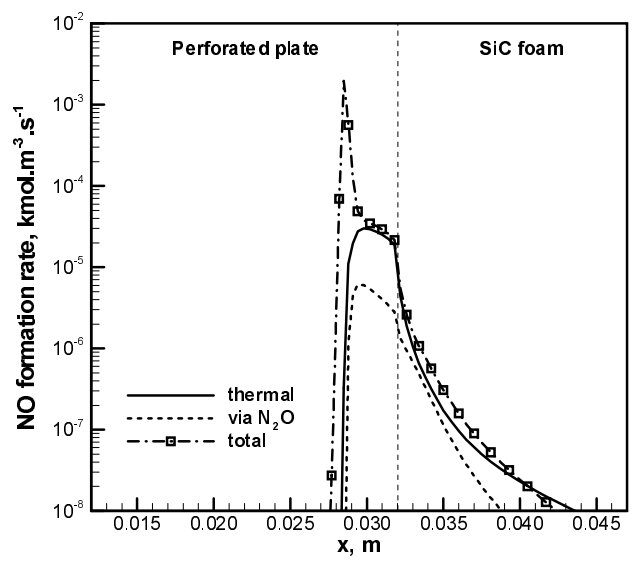

(a)

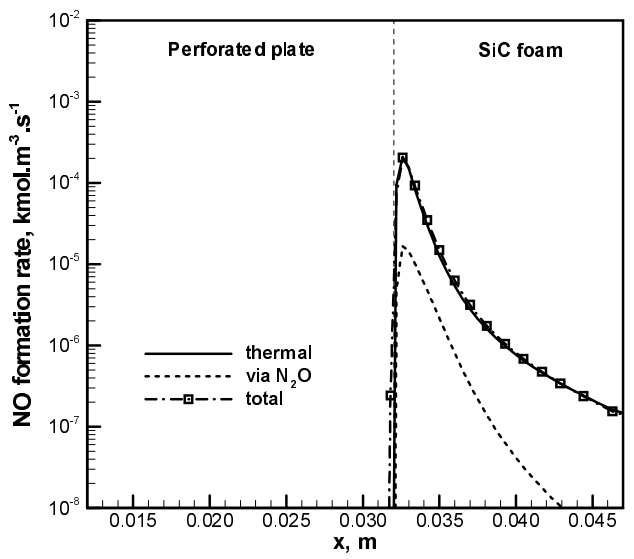

(b)

FIGURE 10 Evolution of NO formation rate along the centerline of a hole for the burner operating at $4 \mathrm{~kW}$ power and $\lambda=1.5$ with (a) perforated plate $\mathrm{PPH} 2$, with the flame front stabilized inside the hole; and (b) perforated plate PPH1.

tion when the flame is anchored in the holes of the perforated plate (as is the case depicted in Fig. 10a), and the corresponding rate could be obtained from the figure by subtraction of the two other contributions from the total. Thus the difference between the total NO formation rate and the sum of the thermal and $\mathrm{N}_{2} \mathrm{O}$-consuming contributions corresponds to the displacement of the equilibrium of the reversible $\mathrm{NO}_{2}$-consuming reaction, equation (6), to the right-hand side:

$$
\mathrm{NO}_{2}+\mathrm{H}=\mathrm{NO}+\mathrm{OH}
$$




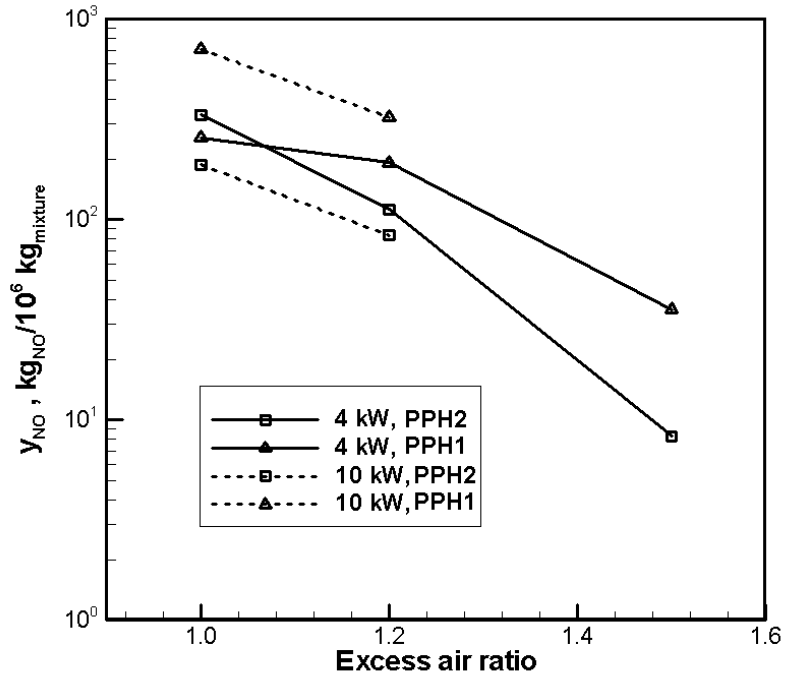

FIGURE 11 Emission of NO as function of the operating conditions and diameter of the holes of the perforated plate.

When the plate with smaller holes is used, the flame front stabilizes in the $\mathrm{SiC}$ foam, and most $\mathrm{NO}$ is formed by means of the thermal mechanism. When the flame is anchored within the holes, the flame temperature is higher, owing to the insulating properties of the perforated plate, leading to formation of NO by an alternative pathway. It is observed in Figure 10a that in the holes, NO is initially formed via the destruction of $\mathrm{NO}_{2}$, which is favored by high temperatures. In the conversion of $\mathrm{NO}_{2}$, both $\mathrm{NO}$ and $\mathrm{OH}$ molecules are formed. Thus the decrease of $\mathrm{NO}_{2}$ and the concomitant increase of available $\mathrm{OH}$ lead to triggering the thermalNO mechanism, which prevails subsequently. In the case of flames stabilized within the pores of the $\mathrm{SiC}$ foam, as is the case illustrated in Figure 10b, the more effective cooling of the combustion products results in lower temperature, and conversion of $\mathrm{NO}_{2}$ to $\mathrm{NO}$ is not favored. This is why, in this case, thermal-NO is the prevailing pathway for formation of this pollutant all along the reaction zone.

The average concentration of $\mathrm{NO}$ at the outlet section of the computational domain is presented in Figure 11 as a function of the operating condition for the burner with the two studied perforated plates. Except for the case with a 4-kW load and an excess air ratio equal to 1.0, it is observed that higher levels of NO emissions are predicted for the burner operating with the plate PPH1, with holes $1 \mathrm{~mm}$ in diameter. This is in agreement with the increase in the solid temperature when this plate is used and with the higher rates of NO formation in the outflow from the burner, as can be observed in Figures 10a and 10b. Even though the emitted NO levels are higher for the PPH1 plate, it is observed that they are af- 


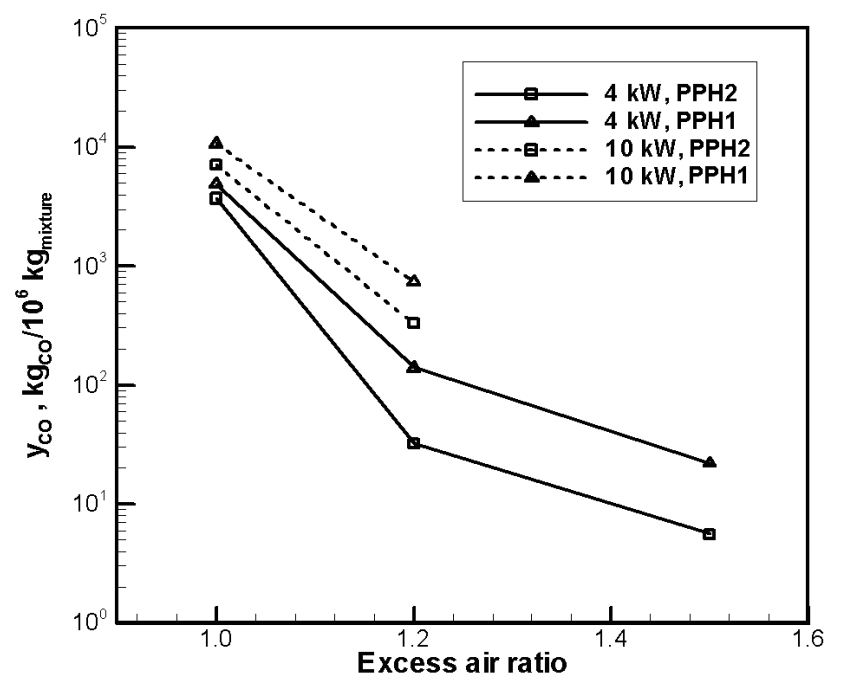

FIGURE 12 Emission of $\mathrm{CO}$ as function of the operating conditions and diameter of the holes of the perforated plate.

fected by the operating conditions in the same way as the temperature. This is due to the prevalence of the thermal-NO mechanism for NO formation when the flame front stabilizes in the $\mathrm{SiC}$ foam.

The predicted emissions of $\mathrm{CO}$ for the two perforated plates are presented in Figure 12. Even though the emissions behavior for this compound does not give any indication of the occurrence of an abnormality in the burner operation, the influence of the hole size on the production of this pollutant is studied. For both plates, $\mathrm{CO}$ emission increases with power, which is due to the reduction of the residence time, since conversion of $\mathrm{CO}$ to $\mathrm{CO}_{2}$ is a relatively slow reaction. In the opposite direction, production of this pollutant is reduced when the excess air ratio increases because the increase of the oxidant agent favors the completion of the combustion process. For all the operating conditions compared in this study, emissions from the burner operating with the PPH1 plate are higher than those from the burner with the PPH2 plate.

\section{CONCLUSION}

The influence of the diameter of the holes of a perforated plate that precedes the flame support layer in a newly designed porous burner was numerically studied by means of a three-dimensional model of a unit cell representative of the geometry of the two-layered porous burner. By means of the finite volume method, the Navier-Stokes equations were solved along with the energy balances for the solid 
and gas phases and the transport equations of the chemical species involved in a skeletal mechanism of reactions. Radiative heat transfer in the porous media was accounted for. Calculations of the burner were performed considering two perforated plates, one of them having holes $2 \mathrm{~mm}$ in diameter (PPH2) and the other with holes $1 \mathrm{~mm}$ in diameter (PPH1), allowing for a discussion of the influence of the diameter of the holes of the perforated plate on the performance of the burner. The main conclusions are listed below.

1. For operating conditions of lower flow velocity the flame front stabilizes within the holes of the perforated plate with larger holes, instead of within the pores of the flame support layer. This is in agreement with the experience of the builders of the burner (Y. Abu-Shareck, personal communication, 2002).

2. A comparison of the performance of the burner using both perforated plates shows that the perforated plate with smaller holes (PPH1) is able to provide the necessary quenching of the reaction front in order that the flame stabilizes within the pores of the $\mathrm{SiC}$ foam layer for all the range of operating conditions.

3. When the 2-mm hole perforated plate is used, the energy released in combustion, for the lower-velocity conditions, occurs inside the holes, yielding a higher peak temperature than with the 1-mm holes. This leads to a too high temperature that damages the perforated plate.

4. The temperatures attained by the gas and solid phases in the SiC foam, for the burner operating with the PPH1 plate, are higher than for the case of the perforated plate with larger holes. This results in a smaller fraction of heat delivered by the burner being in the form of sensible heat of the outflow from the burner.

5. Emissions of pollutants (NO and $\mathrm{CO}$ ) are also higher for the burner operating with the plate with smaller holes. The present results might suggest that usage of a perforated plate with larger-diameter holes could lead to an improved performance of the burner. Nevertheless, it is shown that for safety reasons, the plate with holes $1 \mathrm{~mm}$ in diameter is the appropriate choice.

\section{ACKNOWLEDGMENTS}

This work was partially supported by EC project ENK6-CT-2000-00317. T. C. Hayashi would like to thank the Fundacao CAPES (Brasilia, Brazil) for the Ph.D. fellowship granted.

\section{NOMENCLATURE}

$c_{p, f} \quad$ specific heat of the gas mixture

$\mathcal{D}_{k m} \quad$ molecular diffusivity of the $k$ th species in the mixture

$h_{k} \quad$ molar enthalpy of the $k$ th species 
$h_{v} \quad$ volumetric heat transfer coefficient between the solid matrix and the gas mixture

$k_{f} \quad$ thermal conductivity of the gas mixture

$k_{s} \quad$ thermal conductivity of the porous matrix

$M_{k} \quad$ molecular mass of the $k$ th species

$N_{s p} \quad$ number of chemical species

$p \quad$ pressure

$\mathbf{q}_{r} \quad$ radiative heat flux

$T_{f} \quad$ temperature of the gas mixture

$T_{S} \quad$ temperature of the porous matrix

$\mathbf{v} \quad$ interstitial flow velocity

$y_{k} \quad$ mass fraction of the $k$ th species

$\varepsilon \quad$ porosity of the porous matrix

$\lambda \quad$ excess air ratio

$\mu_{f} \quad$ viscosity of the gas mixture

$\rho_{f} \quad$ density of the gas mixture

$\dot{\omega}_{k} \quad$ molar rate of production of the $k$ th species

\section{REFERENCES}

Barra, A. and Ellzey, J. L. (2004) Heat recirculation and heat transfer in porous burners, Combust. Flame, 137, 230-241.

Barra, A. J., Diepvens, G., Ellzey, J. L. and Henneke, M. (2003) Numerical study of the effects of material properties on flame stabilization in a porous burner, Combust. Flame, 134, 369-379.

Brehmer, T., Heger, F., Lucka, K., von Schloss, J., Abu-Sharekh, Y., Trimis, D., Heeb, A., Kob, G., Hayashi, T. C., Pereira, J. C. F., Founti, M., Kolaitis, D., Molinari, M., Ortona, A., Michel, J.-B. and Theurillat, P. (2003) BIOFLAM project: Application of liquid biofuels in new heating technologies for domestic appliances based on cool flame vaporization and porous medium combustion. In 7th Int. Conf. on Energy for a Clean Environment, IST, Lisbon, CD-ROM, pap. 11.3.

Diamantis, D. J., Mastorakos, E. and Goussis, D. A. (2002) Simulations of premixed combustion in porous media, Combust. Theory Modell., 6, 383-411.

Durst, F. and Trimis, D. (2002) Combustion by free flames versus combustion reactors, Clean Air, 3, $1-20$.

Fiveland, W. A. (1988) Three-dimensional radiative heat transfer solutions by the discrete-ordinates method, AIAA J. Thermophys. Heat Transfer, 2, 309-316.

Gray, W. G. (1975) A derivation of the equations for multi-phase transport, Chem. Eng. Sci., 30, 229-233.

Hayashi, T. C. (2005) Multidimensional modelling and calculation of combustion in porous media. Ph.D. Thesis, Instituto Superior Técnico, Lisbon.

Hayashi, T. C., Malico, I. and Pereira, J. C. F. (2004) Three-dimensional modelling of a two-layer porous burner for household applications, Comput. Struct., 82, 1543-1550. 
Howell, J. R., Hall, M. J. and Ellzey, J. L. (1996) Combustion of hydrocarbon fuels within porous inert media, Prog. Energy Combust. Sci., 22, 121-145.

Hsu, P.-F., Evans, W. D. and Howell, J. R. (1993) Experimental and numerical study of the premixed combustion within nonhomogeneous porous ceramics, Combust. Sci. Technol., 90, 149172.

Kee, R. J., Rupley, F. M. and Miller, J. A. (1995a) CHEMKIN-II: A Fortran chemical kinetics package for the analysis of gas-phase chemical kinetics, rep. SAND89-8009B.UC-706, Sandia National Laboratories, Albuquerque, N. M.

Kee, R. J., Dixon-Lewis, G., Warnatz, J., Coltrin, M. E. and Miller, J. A. (1995b) A Fortran computer package for the evaluation of gas-phase multicomponent transport properties, rep. SAND86-8246.UC-401, Sandia National Laboratories, Albuquerque, N. M.

Kulkarni, M. R. and Peck, R. E. (1992) Analysis of a bilayered porous radiant burner. In 1992 Fall Meeting of the Western States Section of the Combustion Institute, Combust. Inst., Berkeley, CA.

Macdonald, I. F., El-Sayed, M. S., Mow, K. and Dullien, F. A. L. (1979) Flow through porous media - The Ergun equation revisited, Indust. Eng. Chem. Fund., 18, 199-208.

Mital, R., Gore, J. P. and Viskanta, R. (1996) Measurements of radiative properties of cellular ceramics at high temperature, AIAA J. Thermophys. Heat Transfer, 10, 33-38.

Modest, M. F. (1993) Radiative Heat Transfer, McGraw-Hill, Singapore.

Mohammad, A. A., Ramadhyani, S. and Viskanta, R. (1994) Modeling of combustion and heat transfer in a packed bed with embedded coolant tubes, Int. J. Heat Mass Transfer, 37, 11811191.

Özişic, M. N. (1985) Heat Transfer - A Basic Approach, McGraw-Hill, Singapore.

Seshadri, K., Bollig, M. and Peters, N. (1997) Numerical and asymptotic studies of the structure of stoichiometric and lean premixed heptane flames, Combust. Flame, 108, 518-536.

Tong, T. W. and Sathe, S. B. (1991) Heat transfer characteristics of porous radiant burners, ASME J. Heat Transfer, 113, 423-428.

Trimis, D. and Glass, J. (2000) Twelve month report of activities in work package 3 of Bioflam Project, technical report, Thomas Brehmer, OMV, Vienna, Austria.

Tseng, C.-J. (1995) Liquid fuel combustion in porous ceramic burners. Ph.D. Thesis, University of Texas at Austin.

Viskanta, R. and Gore, J. P. (2000) Overview of cellular ceramics based porous radiant burners for supporting combustion, Clean Air, 1, 167-203.

Whitaker, S. (1969) Advances in theory of fluid motion in porous media, Indust. Eng. Chem., 61, $14-28$. 